\title{
USE OF BITOT'S SPOT IN SCREENING FOR VITAMIN A DEFICIENCY IN NIGERIAN CHILDREN
}

\author{
A.I. AJAIYEOBA ${ }^{1 *}$ AND E. SAMAILA ${ }^{2}$ \\ ${ }^{1}$ Department of Ophthalmology, University College Hospital, Ibadan, NIGERIA. \\ ${ }^{2}$ Guinness Eye Centre, Ahmadu Bello University Teaching Hospital, Kaduna, NIGERIA.
}

\begin{abstract}
A total of 2,905 children between 6 and 71 months of age were selected using a 2 stage stratified sampling method and screened for xerophthalmia using World Health Organisation (WHO) criteria and vitamin a deficiency (VAD) using serum retinol < $20 \mu \mathrm{g} / \mathrm{dl}$ (low/marginal). Only 33 cases of xerophthalmia were seen representing a national prevalence of $1.1 \%$. Only 8 children presented with Bitot's spots (XIB) representing $0.3 \%$ of the total number of children screened for marginal VAD and $24.2 \%$ of xerophthalmic cases. Corneal xerosis (stage X2) was the commonest (0.8\%) ocular presentation amongst the total number of children screened and also amongst cases of xerophthalmia where it was responsible for $66.7 \%$. 3 children with VAD (serum retinol < $10 \mu \mathrm{g} / \mathrm{dl}$ ) presented with Bitot's spot, corneal xerosis and corneal scar respectively. All of these 3 children were severely malnourished and sick while one of them was moribund. No cases of conjunctival xerosis (X1A), corneal ulcer (X3A) or keratomalacia (X3B) were encountered in the study. Corneal scars (XS) attributable VAD was responsible to $0.1 \%$. Stages X3A (corneal xerosis) and XS (corneal scars attributable toVAD) of xerophthalmia were above the WHO cut-off points. The national prevalence of marginal VAD defined as serum retinol was $28.1 \%$. Despite its low prevalence, Bitot's spot can be a useful screening tool particularly in a field survey involving a large population of children because of the characteristic cluster distribution of VAD. The implication of this is that a single case of Bitot's spot points to a whole community at risk of VAD.
\end{abstract}

Key words: Bitot's spot, screening, Vitamin A deficiency

${ }^{*}$ Address for correspondence

\section{INTRODUCTION}

Vitamin A deficiency (VAD) is the leading cause of childhood blindness in the world (Sommer et al., 1981) and is a leading cause of morbidity and mortality among pre-school children in developing countries (Beaton et al., 1981). Prevalence of xerophthalmia, the ocular manifestation of VAD, has been considered the primary reference standard for the assessment and determination of VAD as a public health problem (World Health Organisation,1982). Ocular manifestation of VAD has been classified and a detailed description of diagnostic techniques suitable for screening in field surveys including criteria and interpretation is available (Sommer, 1982a). Bitot's spot consists of heaping of desquamated, keratinised epithelial cells, which form a slightly raised area on the conjunctiva.

Bitot's spot, one of the ocular manifestations and most easily recognised, non-blinding signs of VAD usually affecting children aged 3-6 years is a sign of longstanding deficiency, which responds favourably to vitamin $A$ could be a marker to identify those communities with severe and longstanding VAD. Because of the clustering (Sommer, 1982b) nature of $V A D$, identification of a single case of Bitot's spot may signify a community with VAD as a public health problem. It is therefore desirable to evaluate the use of Bitot's spot in screening for VAD in Nigerian children.

\section{MATERIALS AND METHODS}

The survey was a population-based, cross-sectional study, part of a national survey conducted in Nigeria between June and September 1993, designed to estimate the prevalence of VAD and xerophthalmia in the four health zones of Nigeria. Respondents were selected, using a two-stage stratified cluster sampling method.
Ophthalmologists in most areas did Screening and examination. However, in areas where ophthalmologists were not available, ophthalmic residents and nurses did the screening. Both ophthalmic residents and nurses were trained to apply a standardised examination procedure. The screening/examination procedure followed the World Health Organisation (WHO) criteria and staging (Wittpenn et al., 1988) of xerophthalmia for field examination. Ophthalmic examination was carried out with the aid of a pen torch and use of magnification (e.g. loupe). All children's eyes were stained with flourescein dye in order to assess subtle corneal changes, which may be indicative of xerophthalmia. Blood samples obtained from all children were assessed for retinol using the reversed phase, high-pressure liquid chromatography (HPLC) method (Catignani and Bierri, 1983).

Ethical approval for the study was obtained from the Joint Ethical Committee of the College of Medicine, University of Ibadan and the University College Hospital Ibadan, Nigeria. Informed consents were obtained from mothers or guardians after carefully explaining all procedures.

\section{RESULTS}

A total of 2905 children between 6 and 71 months of age were screened for xerophthalmia and VAD. However, data on retinol of some children lacked details or vital information required for meaningful analysis in some aspects of the study. These were largely due to refusal of blood collection, inadequate/undesirable samples (haemolysed), labelling errors, or improper/none entry of vital data. These records were therefore excluded in order to improve the quality of the results. Therefore only the retinol results of 1244 children were reliable for meaningful evaluation. 
Table 1: Prevalence of the various stages of Xerophthalmia in Nigerian children with low/ marginal VAD against the WHO cutoff points.

\begin{tabular}{lrrc}
\hline Stages of Xerophth & Frequency & $\%$ & $\begin{array}{c}\% \text { Indicative } \\
\text { of } \\
\text { health } \\
\text { significance }\end{array}$ \\
\hline No Xerophthalmia & 2872 & 98.8 & $<99$ \\
XIA Conjunct. xerosis & 0 & 0.0 & $>0.5$ \\
XIB Bitot's spot & 8 & 0.3 & $>0.5$ \\
X2 Corneal xerosis & 22 & 0.8 & $>0.01$ \\
X3A/B Corneal ulcer & 0 & 0.0 & $>0.01$ \\
XS Corneal scars & 3 & 0.1 & $>0.05$ \\
\hline TOTAL & 2905 & 100.0 & \\
\hline
\end{tabular}

Table 2: Prevalence of the various stages of Xerophthalmia in Nigerian children with vitamin A deficiency.

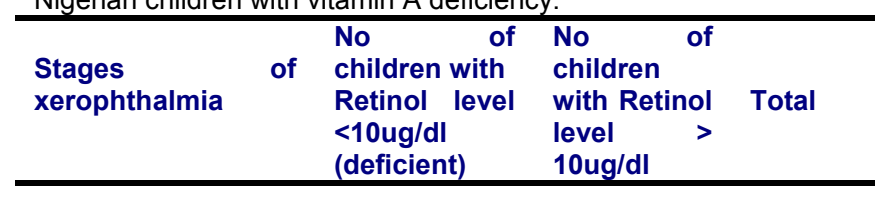

\begin{tabular}{lccc} 
No Xerophthalmia & 98 & 1130 & 1228 \\
XIA Conj. xerosis & 0 & 0 & 0 \\
XIB Bitot spot & 1 & 1 & 2 \\
X2 Corneal xerosis & 1 & 12 & 13 \\
X3A/B Corneal ulcer & 0 & 0 & 0 \\
XS Corneal scars & 1 & 0 & 1 \\
\hline Total & 101 & 1143 & 1244 \\
\hline
\end{tabular}

Only 33 cases of xerophthalmia were seen representing a national prevalence of $1.1 \%$. Only 8 children presented with Bitot's spots (XIB) representing $0.3 \%$ of the total number of children screened for marginal VAD and $24.2 \%$ of xerophthalmic cases. Corneal xerosis (stage X2) was the commonest $(0.8 \%)$ ocular presentation amongst the total number of children screened and also amongst cases of xerophthalmia where it was responsible for $66.7 \%$. No cases of conjunctival xerosis (X1A), corneal ulcer (X3A) or keratomalacia $(\mathrm{X} 3 \mathrm{~B})$ were encountered in the study. Stages X3A (corneal xerosis) and XS (corneal scars attributable toVAD) of Xerophthalmia were above the WHO cut-off points. The prevalence of the various stages of xerophthalmia in children with low/marginal VAD $(<20 \mathrm{ug} / \mathrm{dl})$, against the WHO cut-off points is shown in Table 1. 3 children with VAD (serum retinal < $10 \mu \mathrm{g} / \mathrm{dl}$ ) presented with Bitot' spot, corneal xerosis and corneal scar respectively. All the 3 children were severely malnourished and sick while 1 was moribund.
Table 2 shows the prevalence of the various stages of xerophthalmia in children with VAD (retinol $<10$ and $>$ $10 \mathrm{ug} / \mathrm{dl}$ ).

The national prevalence of marginal vitamin $A$ deficiency (defined by serum retinol $<20 \mu \mathrm{g} / \mathrm{dl}$ or 0.7 $\mu \mathrm{mol} / \mathrm{l})$ was $28.1 \%$ (95\% confidence interval $25.4-$ $31.0 \%)$ and severe retinol deficiency $(10 \mu \mathrm{g} / \mathrm{dl}$ or 0.35 $\mu \mathrm{mol} / \mathrm{l}$ ) was $7.0 \%$ (95\% confidence interval $5.5-8.8 \%$ ). The distribution curve was positively skewed and differed significantly from the normal (Gaussian) pattern. The values ranged from 5 to $134 \mathrm{ug} / \mathrm{dl}$. The national mean serum retinol value was $31.8 \mathrm{ug} / \mathrm{dl}$. $(\mathrm{SD}=18.6)$.

\section{DISCUSSION}

Xerophthalmia is the most readily recognized clinical manifestation of Vitamin A deficiency (VAD) and has been the most widely employed, definitive criteria for accessing weather Vitamin A poses a significance public health problem. Xerophthalmia occurs when serum levels drop below $20 \mu \mathrm{g} / \mathrm{dl}$, and is more likely when levels are below $10 \mu \mathrm{g} / \mathrm{dl}$. Many of the eye signs of VAD are pathognomonic for the condition and so can be used to diagnose and screen VAD. However not all children who were deficient developed xerophthalmia (i.e. they were sub clinically deficient). This goes to show that children who are clinically deficient and by definition have xerophthalmia as well as those who are sub clinically deficient and but do not develop xerophthalmia may both be vitamin A deficient. It therefore means that those with xerophthalmia therefore only represent the tip of the iceberg of those who are deficient in the community. However it has been documented that children with clinical deficiency as well as those with sub-clinical deficiency are at increased risk of dying (Sommer et al.,1983). It is therefore important to have a sensitive marker like Bitot's spot, which will lead us to that community

When bitot's spot is seen in children over the age of 6 years, it may signify a previous early Vitamin $A$ deficiency and may therefore resemble cornea scars (XS). Both bitot's spots and cornea scars are reminiscences of early Vitamin A deficiency but differ from each other in that, whereas bitot spot is specific for Vitamin A deficiency, cornea scars may be due to other causes like trauma. Some workers (Mclaren, 1980) have suggested the role of other ultra violet rays in particular in the genesis of bitot's spot, which it is usually located in the exposed part of the conjunctiva.

Our finding in this study, of a low prevalence of xerophthalmia including bitot's spot despite the large number of children screened has been the experience of other workers as well, and has been documented (Sommer, 1982b). This low prevalence might be due to the fact that ocular manifestation of xrophthalmia represents a long term of severe and chronic effect of VAD on a target organ like the eye. Before the manifestation of xerophthalmia, children must have suffered tremendously from the systemic complications of $\mathrm{VAD}$, which more often than not might have claimed the lives of these children. It was therefore not surprising that all the children with VAD presenting with xerophthalmia were sick children. Even though bitot spot is a non-blinding condition, it is known (Sommer, 1983) that children with bitot's spot have a mortality rate which is 6.6 times greater than children without it. In as 
much as we know that bitot's spot is specific for Vitamin A deficiency particularly when seen in children under 6 years of age and because of the clustering nature of Vitamin A deficiency, it can be inferred that a single case of bitot's spot might signify a community with vitamin A deficiency. However it would have been desirable to look at a larger sample of children.

In conclusion, as has been shown that children who are clinically deficient as well as those who are sub clinically deficient are at increased risk of dying,(Sommer,1983) it is therefore important to have a sensitive marker like Bitot's spot which will lead us to such community whose young generation may have been afflicted by a silent killer. The overall health implication of this cannot be overemphasised.

\section{REFERENCES}

Beaton GH, Martorell R, L'Abbe KA, Edmonston B, McCabe G, Ross AC, et al. (1981). Effectiveness of vitamin A supplementation in the control of young child morbidity and mortality in developing countries. Toronto. University of Toronto. Oxford University Press.

Catignani GL, Bierri JG (1983). High Pressure Liquid Chromatography. Clin. Chem. 29:708-712.

McLaren DS (1980). Nutritional Ophthalmology. Academic

Press, 1980. London.

Sommer A (1982a). Field guide to detection and control of xerophthalmia. $2^{\text {nd }}$ edition. Geneva. WHO.

Sommer A (1982b) Nutritional blindness. Xerophthalmia and keratomalacia. New York

Sommer A, Tarwotjio I, Hussaini G (1983). Increased mortality in children with mild Vitamin A deficiency. Lancet 2 : $585-588$

Sommer A, Tarwotjo I, Hussaini G, Susanto D, Soegiharto $T$ (1981). Incidence, prevalence and scale of blinding malnutrition. Lancet. 1:1407-8.

Wittpenn JR, West KP, Keenum D (1988). ICEPO Training Manual. Assessments of Vitamin A status by impression cytology. The John Hopkins University, Baltimore.

World Health Organisation (1982). Control of vitaminA deficiency and xerophthalmia. Technical Report Series No. 672. Geneva. WHO.

Received: January 2001

Accepted: September 2001 
